\title{
Overview of the Electromagnetic Calorimeter Trigger system at the Belle II Experiment
}

\section{S.H. Kim*}

Dept. of Physics, Hanyang University

E-mail: sunghyun.kimabel le2 . org

\section{I.S. Lee}

Dept. of Physics, Hanyang University

E-mail: is lee@hep.hanyang.ac.kr

\section{C.H. Kim}

Dept. of Physics, Hanyang University

E-mail: chkim@hep.hanyang.ac.kr

\section{Y.J. Kim}

Dept. of Physics, Korea University

E-mail: rladudwns118@korea.ac.kr

\section{Y. Unno}

Dept. of Physics, Hanyang University

E-mail: yunnolpost.kek.jp

\section{B.G. Cheon}

Dept. of Physics, Hanyang University

E-mail: bgcheon@hanyang.ac.kr

The Belle II at the SuperKEKB collider in Japan has been constructed toward a physics run in early of 2018 with an ultimate target of 40 times higher instantaneous luminosity than the KEKB collider, which was $2.1 \times 10^{34} \mathrm{~cm}^{-2} \mathrm{~s}^{-1}$. The main physics motivation is to search for the New Physcis from heavy quark/lepton flavor decays. We have upgraded the Electromagnetic Calorimeter(ECL) hardware trigger system in order to select an event of interest efficiently under much higher luminosity and beam background environment than the KEKB. ECL trigger logic based on two main triggers, the total energy and the number of clusters, would be improved with an FPGA-based flexible architecture and a high speed serial link for the data transfer. In this report, progress of the ECL trigger system development will be outlined and preliminary results from beam collision data in Phase II run will be described.

ICHEP 2018, International Conference on High Energy Physics

4-11 July 2018

Seoul, Korea

${ }^{*}$ Speaker. 


\section{Introduction}

The Belle experiment [1] at KEK in Japan took 772 million pairs of $B$ - and $\bar{B}$-mesons produced by the KEKB accelerator [2] to study CP violations in the neutral $B$-meson system. Most of the results are in good agreement with the Standard Model (SM) predictions. The SuperKEKB accelerator [3] and the Belle II are expected to find new physics beyond SM in flavor physics.

The total cross sections and trigger rate of physical processes at the target luminosity of $8 \times$ $10^{35} \mathrm{~cm}^{-2} \mathrm{~s}^{-1}$, which is 40 times higher than the peak value of the KEKB collider.

\section{ECL Trigger System}

$8763 \mathrm{CsI}(\mathrm{Tl})$ scintillation crystals have been selected for the ECL detector. The data of energy deposition in crystal counter is sent to ShaperDSP, then 16 fast-shaping signals from neighboring 4 $\times 4$ crystals are merged in the fast shaper circuit. This is implemented on the main shaper board to form an analog trigger sum called the trigger cell (TC), which is a basic unit in ECL trigger system and 576 TCs used in total.

FADC Analysis Module (FAM) received the analog TC data and digitize it. To measure energy and decide timing, a wave form analysis in FPGA is performed by $\chi^{2}$ fit on FPGA. All FAMs send the TC energy and timing data to ECL Trigger Master (ETM) via the GTX serial link. ETM generates phsysics and Bhabha trigger signals in the FPGA.

Physics trigger conditions are as following : (1) Total energy trigger $\left(E_{t o t}\right)$ is energy sum of barrel and forward endcap excluding most inner layer greater than $1 \mathrm{GeV}$. (2) The number of isolated clusters (ICN), which is to count the number of particle clusters that deposit the energy in ECL, is greater than 3. When an event, which is not tagged by Bhabha trigger condition, does satisfy either (1) or (2), the ETM generates physics trigger signal. The 3-Dim ( $\mathrm{r}-\boldsymbol{\theta}-\phi)$ back-to-back topology is used for Bhabha veto trigger to avoid any misidentification of low multiplicity process such as $\tau$ or Initial State Radiation (ISR) events as a Bhabha event. After the ECL trigger final decision, the ETM sends ECL-TRG informations to GDL and GRL for further trigger decision together with other sub-triggers. For the offline analysis, the ETM sends all the TC informations to the DAQ by receiving the trigger signal from GDL.

\section{Conclusion}

All of ECL trigger hardwares have been successfully installed and tested. We prepare 3-Dim Bhabha veto logic, which is first used in Belle II. All ECL trigger hardware, firmware and softwares are successfully worked at Phase-II run in Belle II experiment.

\section{References}

[1] A. Abashian et al. (Belle Collaboration), The Belle detector, NIM A, 479 (117-232) [2002]

[2] S. Kurokawa, E. Kiktani, Overview of the KEKB accelerators, NIM. A, 499 (1) [2003]

[3] Y. Funakoshi, Crab Crossing Scheme at KEKB, Beam Dynam. Newslett, 40 (27) [2006]

[4] Y. Iwasaki et al., Level 1 Trigger System for the Belle II experiment, IEEE Trans. Nucl. Sci, 58 (1807-1815) [2011] 